\title{
Material Aspects for preparing HTS Quasiparticle Injection Devices
}

\author{
C. W. Schneider*, R. Moerman, F. J. G. Roesthuis, R. G. Wichern, G. J. Gerritsma and H. Rogalla, \\ University of Twente, Dept. of Applied Physics, Enschede, The Netherlands
}

\begin{abstract}
Quasiparticle (QP) injection devices based on HTS could play an important role in future superconducting applications if material aspects can be better controlled. One reason why this kind of device received little attention in the past is the lack of an appropriate barrier for $Q P$ tunnelling. In a series of experiments, we used different barriers to test if they are suitable, $i$. e. if a current and possibly a voltage gain can be achieved. We improved the performance of planar YBCO/natural barrier/Au devices and a current gain of more than 6 at $40 \mathrm{~K}$ was observed. Most devices, however, showed signs of heating effects. Another barrier material was $\mathrm{SrTiO}_{3}$ with layers of 5 - $6 \mathrm{~nm}$ thickness. Current-voltage characteristics showed that the barriers were continuous and we observed current gains of up to 3 at $60 \mathrm{~K} . \mathrm{PrBa}_{2} \mathrm{Cu}_{3} \mathrm{O}_{7-x}$ is an interesting candidate if one could overcome the problem of resonant inelastic tunnelling for QP. In a series of experiments we demonstrated that, even for $3 \mathrm{~nm}$ thin PBCO barriers on a- and c-axis oriented $\mathrm{YBa}_{2} \mathrm{Cu}_{3} \mathrm{O}_{7-\mathrm{x}}$, most devices showed at best a current gain of 1 . However, we have indications that a current gain of 10 could be possible with unity voltage gain.
\end{abstract}

\section{INTRODUCTION}

The family of quasiparticle injection devices could be an interesting candidate for applications where the current of a device needs to be controlled via a third terminal. There, one does not necessarily need current or voltage gain, whereas for digital application a power gain is the basis for any kind of transistor. An essential requirement for the progress of preparing and successfully implementing quasiparticle injection devices using high temperature superconductors (HTS) such as $\mathrm{YBa}_{2} \mathrm{CuO}_{7-x}$ (YBCO) is the availability of suitable tunnelling barriers permitting direct or elastic resonant tunnelling of quasiparticles which will ensure a low power consumption for the injection terminal. For low- $T_{c}$ materials, tunnelling barriers are well established [1-3]. But for today's HTS devices in general, such kind of barriers are still missing. Some barriers used for low- $T_{c}$ devices have the advantage that one can grow fully epitaxial planar devices which is also a preferred option for a future HTS device technology.

In this paper we report recent investigations of planar $\mathrm{YBCO} / \mathrm{barrier} / \mathrm{Au}$ devices where a number of different barrier

\footnotetext{
The manuscript was received Aug 26, 1996

This work was financially support by the EU (ESPRIT project number 8132 and by Human Capital and Mobility (WELITTD-HTS, Contract number ERB CHRX-CT94-0523).

*Present Address: Forschungszentrum Karisruhe, INFP, P.O. Box 3640 , 76021 Karlsruhe, Germany
}

materials were studied, some of which can be grown epitaxially on YBCO. The current gain of these devices was measured as a function of temperature between $T_{c}$ and $10 \mathrm{~K}$, and results indicate that a current gain of 10 is possible even at operating temperatures of $60 \mathrm{~K}$ or higher.

\section{DEVICE STRUCTURE}

For the experiments described in this paper, a planar superconductor/barrier/Au structure was used (Fig. 1) as described previously [4-6], with YBCO or DyBCO as the superconductor. Quasiparticles are injected via a barrier into a $2-5 \times 20 \mu \mathrm{m}^{2}$ wide YBCO bridge using a Au counter electrode. The thickness of the superconductor varies between 40 and $80 \mathrm{~nm}$ and the thickness of the Au electrode is $50 \mathrm{~nm}$. Due to a large specific resistance of the injector contact $\left(\mathrm{R}_{\mathrm{n}} \mathrm{A}>10^{-5} \Omega \mathrm{cm}^{2}\right.$ for $\mathrm{YBCO} / \mathrm{Au}$ contacts $)$, the injected quasiparticle flow is uniform over the contact area. To insulate the injection electrode and the wiring from the base electrode we used polycrystalline PBCO.

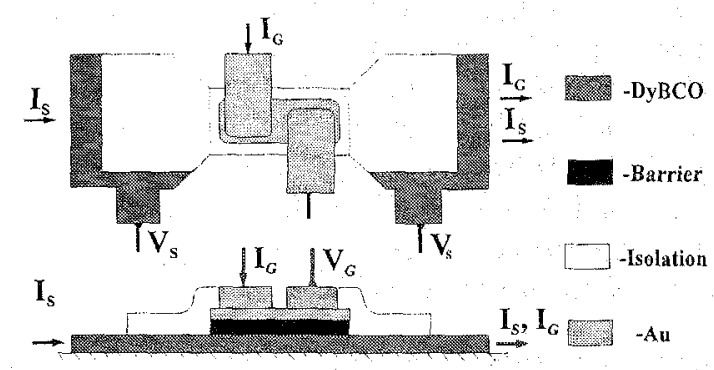

Fig 1: Schematic top and cross sectional view of the quasiparticle injection device.

To pattern (100) films into the planar injection device structure reliably, we applied an inhibit growth technology called selective epitaxial growth (SEG) by sputtering a thin layer of Ti onto the substrate. After the YBCO deposition, a thin layer of $\mathrm{Au}$ is sputtered to protect the (100) surface in order to ensure further successful processing of the device.

\section{EXPERIMENTAL}

The Current-voltage Characteristic (IVC) of the superconducting bridge under quasiparticle injection is measured using a four point configuration. Modulating the critical current $I_{c}$ of the superconductor with the injection current $I_{G}$ leads to an asymmetry in the IVC which is 
independent of the direction of the currents. The reasons for the observed asymmetry are twofold: firstly, a summation of $I_{G}$ and $I_{c}$ called current summation effect and secondly, an additional non-linear suppression of $I_{c}$ due to a nonequilibrium energy distribution of the injected quasiparticles. The current summation effect yields an almost linear suppression of $I_{\mathfrak{c}}$ with $I_{G}$ flowing parallel to $I_{c}$ and no suppression if $I_{G}$ and $I_{c}$ flow anti-parallel. A non-linear suppression of $I_{c}$ with $I_{G}$ flowing parallel is generally attributed to a non-equilibrium effect due to an excess density of quasiparticles and their non-equilibrium energy distribution. If the non-equilibrium relaxation of quasiparticles is dominant, the asymmetry in the IVC will be less pronounced. For $I_{c}$ flowing parallel to $I_{G}, I_{c}$ cannot be suppressed completely due to the still present current summation effect.

The current and voltage gain for quasiparticle devices is defined as $\mathrm{K}_{\mathrm{C}}=\Delta \mathrm{I}_{\mathrm{c}} / \Delta \mathrm{I}_{\mathrm{G}}$ and $\mathrm{K}_{\mathrm{V}}=\mathrm{V}_{\text {out }}\left(\mathrm{I}_{\mathrm{c}}\right) / \mathrm{V}_{\mathrm{G}}\left(\mathrm{I}_{\mathrm{G}}\right)$ respectively with the output voltage $V_{\text {out }}=\rho \mathrm{J}_{c} \ell$ in the dissipative state $(\rho$, the resistivity, $\mathrm{J}_{\mathfrak{c}}$, the current density and $\ell$, the length of the track). For a current gain $\mathrm{K}_{\mathrm{C}} \leq 1$, the current summation effect is dominant and if $K_{C}>1$, the non-equilibrium contribution is dominant. One experimental problem with injection devices is to distinguish between a thermal effect and effects originating through the non-equilibrium state. To see more clearly the non-equilibrium effect, it is better to work with short superconducting bridges to remove heat more efficiently. For long tracks it will take time to remove heat produced by the dissipated power in the injection terminal. However, a shortcoming of using short bridges is a decreased chance to obtain a voltage gain bigger than 1 .

\section{BARRIER MATERIALS}

The choice of barrier material for injection devices is of crucial importance. Early experiments used HTS MgO tunnel barriers $[7,8]$. Due to a very high barrier potential of $2.8 \mathrm{eV}$, a $1-2 \mathrm{~nm}$ continuous $\mathrm{MgO}$ tunnelling barrier on a $\mathrm{YBCO}$ surface is required, but the average surface roughness for YBCO films is usually much bigger. The current gain reported was about 1.6 at $4.2 \mathrm{~K}$. Another attempt used a naturally-grown barrier on (001) oriented $\mathrm{YBCO}[4,5]$; at first a current gain of 2 at $60 \mathrm{~K}$ and later of more than 6 at $40 \mathrm{~K}$ [9] was achieved. The other barrier material tried was PBCO, with the barrier thickness varied between 10 and $40 \mathrm{~nm}$. Despite very good lattice matching and epitaxial growth on YBCO a current gain $K_{C} \leq 1$ was obtained [4,5]. The reason for $\mathrm{K}_{\mathrm{C}}<1$ are probably localised states in the thin PBCO layer, resulting in the dominance of inelastic tunnelling effects. This, in turn, leads to a loss of injection energy in the barrier.

For the experiments presented in this paper we used a naturally grown YBCO barrier, called natural barrier, on (001) and (100)-oriented YBCO and DyBCO. The superconducting film is prepared in one vacuum system and the deposition of the $\mathrm{Au}$ counter electrode takes place in a separate vacuum chamber. In between, the YBCO surface is exposed to air. The other barriers studied were in-situ deposited $\mathrm{Nb}$-doped $\mathrm{SrTiO}_{3}(0.5 \mathrm{wt} \% \mathrm{Nb})$ and $\mathrm{SrTiO}_{3}$, using pulsed laser ablation (PLD) from a single crystal target on (001)-oriented YBCO and in-situ-deposited PBCO and $\mathrm{Ga}$ doped PBCO (nominally $70 \% \mathrm{Ga}$ doping) using off-axis RF sputtering and PLD. The thickness of the PBCO and Gadoped PBCO varies between 3 to $5 \mathrm{~nm}$ on (001) as well as (100) oriented YBCO and DyBCO and the thickness for the STO barriers is $5-6 \mathrm{~nm}$. YBCO and DyBCO films used to grow the barriers where optimised towards no or very little outgrowth over the entire chip area $(5 \mathrm{~mm} \times 10 \mathrm{~mm})$ and the average roughness varies between 3 to $5 \mathrm{~nm}$ peak-to-peak, as measured by atomic force microscopy. This ensures that even very thin barriers can be grown pinhole free.

\section{DISCUSSION}

In the following paragraphs, we would like to discuss some properties of the injection devices with different barrier materials.

\section{A. Natural Barrier}

Depending on the preparation conditions for the barrier, we are able to vary the junction resistance from a few ohms to several $10 \mathrm{k} \Omega$. With increasing junction resistance, we note an improvement in the current gain from 2 at $60 \mathrm{~K}$ to 6.2 at $40 \mathrm{~K}$. For injection devices with the highest barrier resistance, we also noticed that with decreasing temperature the junction resistance increases slightly. This was not observed for injection barriers with low resistance, which suggests that the high resistive natural barriers used for the injection experiments are more likely to be a mixture of natural barrier and possibly a cubic YBCO phase. The latter conclusion originates from work on ramp-type junctions, where a layer of cubic YBCO on the ramp was observed using TEM after ion beam milling [10].

A current gain of 6.2 at $40 \mathrm{~K}$ (see Figure 2) corresponds to a critical current of $27 \mathrm{~mA}$ for the superconducting bridge which is controlled by an injection current of about $4.3 \mathrm{~mA}$, suppressing $I_{c}$ completely. This demonstrates that a reasonably large current can be controlled efficiently by a smaller current. The temperature dependence for the current gain in Figure 2 shows a saturation with decreasing temperature which is attributed to induced heating effects. A direct proof for heating effects is also shown in Figure 2 (dotted curve, STO53). Here, with decreasing temperature the current gain is starting to decrease having already reached a current gain of 5.5 at $63 \mathrm{~K}$. From this curve it is possible to estimate a lower limit of the actual injected power by calculating the voltage across the $\mathrm{YBCO} / \mathrm{Au}$ interface. A power density bigger than $3700 \mathrm{~W} / \mathrm{cm}^{2}$ can be considered as a lower limit where thermal effects will be dominant in the injection device [11]. Knowing the device area and $I_{G}$ we estimate $V_{G} \geq 0.5 \mathrm{~V}$ corresponding to a minimum for the dissipated power of $2 \mathrm{~mW}$. The local temperature rise in the 
superconducting bridge due to the dissipated power amounts to more than $3 \mathrm{~K}$ as estimated from a thin slab model [12]. This leads to the conclusion of heating effects being present in the device and subsequently reducing the measured current gain.

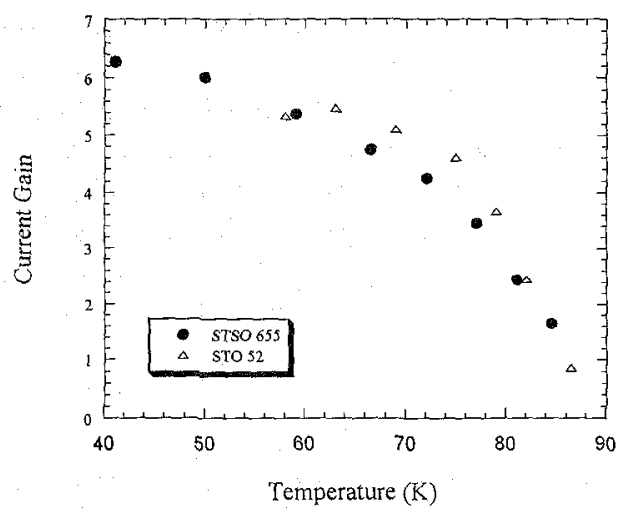

Fig 2: Current gain vs. Temperature for two different devices.

A number of injection devices with natural barriers on (100) YBCO films showed no current gain because the barrier resistance was too low, typically a few ohms over the injection area. The way we prepare natural barriers on (001) oriented YBCO gave reproducible results, indicating that the naturally higher resistance of the $\mathrm{YBCO} c$-axis could also play a significant role for the observed current gain, placing some question marks on the realisation of (100) oriented devices.

\section{B. $\mathrm{SrTiO}_{3}$ and $\mathrm{Nb}$-doped $\mathrm{SrTiO}_{3}$ Barriers}

Thin $\mathrm{SrTiO}_{3}$ barriers, $5-6 \mathrm{~nm}$ thick, are prepared in-situ on very smooth $50-80 \mathrm{~nm}$ thick YBCO films. The measured barrier resistance of $\geq 1 \mathrm{M} \Omega$ of several devices on chip as well as chip-to-chip demonstrates that these barriers must be continuous. The same was true for $\mathrm{Nb}$-doped $\mathrm{SrTiO}_{3}$ barriers suggesting that it is not possible to prepare these very thin doped barriers. For some devices, we measured a current gain; the best result was a current gain of 3 at $64 \mathrm{~K}$. No attempt was made to prepare thinner $\mathrm{SrTiO}_{3}$ barriers, but we would expect that continuous barriers down to $3 \mathrm{~nm}$ are feasible. There is some hope that this type of barrier could show true QP tunnelling and for this reason it is worthwhile to investigate thin $\mathrm{SrTiO}_{3}$ further.

\section{C. $P B C O$ and $G a$-doped $P B C O$ Barrier}

Recent experimental and theoretical work on PBCO [13] presented convincing evidence that a resonant tunnelling channel is responsible for the transmission of QP through PBCO barrier. If one could prepare continuous PBCO barriers thinner than the average hopping length of about $6-8$ $\mathrm{nm}$ for QP [14], direct tunnelling could be possible. An improvement for PBCO barriers should be Ga-doping of
PBCO because Ga fills localised states in the PBCO and therefore increases direct tunnelling for Cooper pairs. This was shown for ramp-type junctions with Ga-doped $\mathrm{PBCO}$ as barrier, where a $I_{c} R_{n}$ value of $10 \mathrm{mV}$ was reported [15].

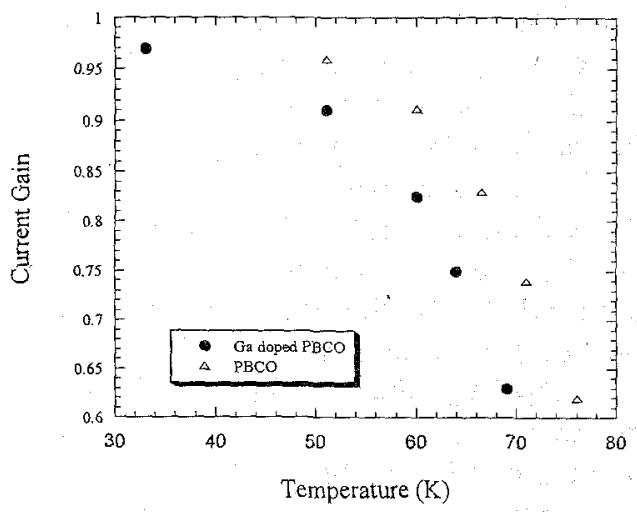

Fig. 3: Current gain as function of temperature for injection device with $\mathrm{Ga}$ doped PBCO (open circles) and PBCO barriers (filled quares).

For the conducted experiments the barrier resistance for $5 \mathrm{~nm}$ thick PBCO is of the order $\mathrm{k} \Omega$ and increases with decreasing temperature. The high resistance and the temperature dependence of the barrier resistance indicates a well defined PBCO barrier. For the Ga-doped PBCO, the barrier resistance below $T_{c}$ was about $5 \mathrm{k} \Omega$ and increased to approximately $15 \mathrm{k} \Omega$. For all devices using PBCO on (001)oriented YBCO, the current gain was less than one at the lowest temperature measured. The same was observed for a $3 \mathrm{~nm}$ thick PBCO barrier on (100) YBCO and for $5 \mathrm{~nm}$ thick Ga-doped PBCO barriers on (001)-oriented YBCO (see Figure 4).

A conclusion of these experiments is that, for very thin PBCO or Ga-doped PBCO barriers, a current gain of the order 1 can be achieved and it is unlikely to obtain better results even for even thinner barriers. Therefore, resonant inelastic tunnelling for QP via localised states cannot be overcome easily and even the partial filling of these states using Ga does not allow a true tunnelling barrier for QP. The saturation of the current gain with decreasing temperature indicates that, as for the natural barrier, some heating is present. One more indication of the ineffectiveness of $\mathrm{PBCO}$ as QP injection barrier is shown in Figure 4. Here, $I_{c}$ is plotted vs. $I_{G}$ at $51 \mathrm{~K}$. A linear suppression of $I_{c}$ for $I_{G}$ flowing parallel to $I_{c}$ and little suppression of $I_{c}$ for $I_{G}$ flowing anti-parallel suggests that the main effect on $I_{c}$ originates from the current summation effect, with some components of a non-equilibrium state present. The only plausible explanation for the current summation effect is if the energy to inject $\mathrm{QP}$ into the superconductor is consumed in the barrier leaving little energy left to break Cooper pairs into QP which could recombine via the non-equilibrium state. 


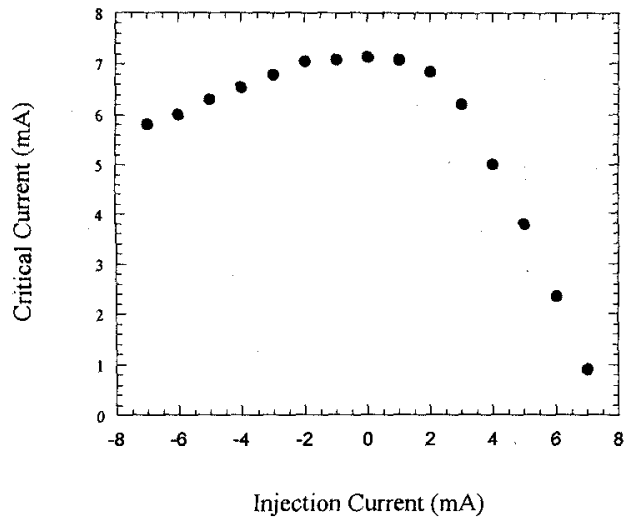

Fig. 4: $I_{c}$ vs. $I_{G}$ for $\mathrm{YBCO} / \mathrm{PBCO} / \mathrm{Au}$ injection device.

However, we have some indication that a current gain of 10 or more at technically interesting temperatures using an appropriate barrier material is possible. For one experiment we prepared four $\mathrm{DyBCO} / \mathrm{PBCO} / \mathrm{Au}$ injection devices with $5 \mathrm{~nm}$ PBCO on $80 \mathrm{~nm}$ DyBCO. Two devices could not be measured. For the third device, we measured a current gain of 3 at $60 \mathrm{~K}$ and for the fourth device a current gain of 9.2 at $60 \mathrm{~K}$ as shown in Figure 5. This is the biggest current gain measured so far meaning that a critical current of $35 \mathrm{~mA}$ was controlled by an injection current of $3.75 \mathrm{~mA}$. Current biasing the superconducting bridge at $I_{c}$ and measuring the voltage drop under current injection, we observed a large voltage of the order $1 \mathrm{~V}$ combined with a thermal hysteresis. This suggests that even for the very short superconducting bridges used for these experiments, voltage gain and hence power gain, is possible.

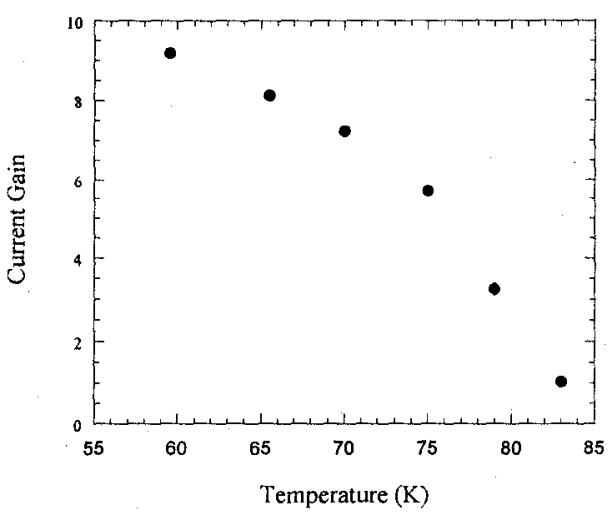

Fig. 5: $\mathrm{K}_{\mathrm{C}}$ as function of temperature for a DyBCO/PBCO/Au injection device. The biggest current gain obtained was 9.2 at $60 \mathrm{~K}$.

\section{CONCLUSION}

We demonstrated that for different types of barriers current gain can be achieved. Experiments using a natural YBCO barrier are promising with the highest current gain of 6.2 at $40 \mathrm{~K}$. The main problems for injection devices are heating effects caused by the very high injection power of several $\mathrm{mW}$. Very thin $\mathrm{SrTiO}_{3}$ barriers show a current gain of 3 at $60 \mathrm{~K}$ and if it is possible to prepare $1-3 \mathrm{~nm}$ thin continuous $\mathrm{SrTiO}_{3}$ barriers, interesting devices might be possible. Thin barriers of PBCO and Ga doped PBCO are interesting due to the very good lattice match to YBCO. No current gain bigger than 1 was observed, even for $3 \mathrm{~nm}$ thick PBCO on (100)oriented YBCO. The most likely explanation is inelastic resonant tunnelling through localised states for QP consuming most of the injection energy in the barrier. Ga-doping of PBCO to fill localised states in the barrier gave no improvement and no current gain was observed. Finally, we have evidence that a current gain of 10 is possible at technologically interesting temperatures. By optimising the devices, a unity voltage gain seems possible.

\section{REFERENCES}

[1] H.D. Leduc, A. Judas, S.R. Cypher et al., "Submicron Area $\mathrm{NbN} / \mathrm{MgO} / \mathrm{NbN}$ Tunnel Junctions for SIS Mixer Application," IEEE Trans. Appl. Supercond. vol. 27, p. 3192 Feb. 1991.

[2] Z. Wang, A. Kawakami, Y. Uzawa et al., "High critical current density NbN/AIN/NbN tunnel junctions fabricated at ambient temperature MgO substrates," App. Phys. Letts., vol. 64, pp. 2034-2036, 1994.

[3] Z.H. Barber, D.M. Tricker, and M.G. Blamire, "The Fabrication and Characterisation of NbCN/AIN Heterostructures," IEEE Trans. Appl. Supercond., vol. 5, p. 2314, Feb. 1995

[4] Yu.M. Boguslavskij, K. Joosse, F.J.G. Roesthuis et al. "Nonequilibrium effect under quasiparticle injection in YBCO-based three-terminal devices at high operating temperatures," Conference proceedings of the EUCAS '93, Göttingen, Germany, 1993

[5] Koen Joosse, "High $T_{c}$ Superconducting Three Terminal Devices," PhD thesis, University of Twente, 1994.

[6] Yu.M. Boguslavskij, K. Joosse, F.J.G. Roesthuis et al,, "Quasiparticleinjection effect in $\mathrm{YBa}_{2} \mathrm{Cu}_{3} \mathrm{O}_{7-\mathrm{x}}$-base planar structures," Physica $B$ vol. $194-196$, pp. $85-86,1994$

[7] Ienari Iguchi, Kiejin Lee, and Kazuhiro Nukui, "Quasiparticle Injected $\mathrm{YBa}_{2} \mathrm{Cu}_{3} \mathrm{O}_{7-y}$ Three Terminal Devices," Advances in Superconductivity $V I, 1993$.

[8] Quian Wang and Ienari Iguchi, "Effective suppression and enhancement of critical current in a non-equilibrium $\mathrm{YBa}_{2} \mathrm{Cu}_{3} \mathrm{O}_{7-x}$ film due to tunnel injection of quasiparticles," Physica $C$, vol. 228, pp. $393-400,1994$

[9] C.W. Schneider, R. Moerman, F.J.G. Roesthuis et al., "Current Gain using High $\mathrm{T}_{\mathfrak{c}}$ Three Terminal Devices," Inst. Phys. Conf. Ser., vol. 148 , pp. 1677 - 1680, 1995.

[10] C.L. Jia, M.I. Faley, U. Poppe et al., Appl. Phys. Letts., vol. 59, p 982, 1995.

[11] L. Shi, G.L. Huang, C. Lehane et al., "Study of photoresponse of high $\mathrm{T}_{\mathrm{c}} \mathrm{Y}-\mathrm{Ba}-\mathrm{Cu}-\mathrm{O}$ ultrathin films using picosecond laser pulse train," Appl. Phys. Letts. vol. 63, pp. 2830-2832, 1993.

[12] C.W. Schneider, G.J. Gerritsma, and H. Rogalla, "Injection and three terminal Devices," Proceedings of the $2^{\text {ind }}$ Workshop on HTS Applications and New Materials, Twente University, 1995.

[13] I.A. Devyatov and M. Yu. Kuprianov, JETP Letts. vol. 59, p 200 , 1994.

[14] G.K. van Ancum, "Electronic Transport Properties of $\mathrm{PrBa}_{2} \mathrm{Cu}_{3} \mathrm{O}_{7-d}$ " $\mathrm{Ph} . \mathrm{D}$. Thesis, University of Twente, 1995.

[15] M.A.J. Verhoeven, G.J. Gerritsma, and A. A. Gulubov, "Ramp Type HTS Josephson Junctions with PrBaCuGaO Barriers," IEEE Trans. Appl. Supercond., vol. 5, p. 2095. March 1995. 